\title{
The Responses of Pineapple Varieties to Forced-Flower Treatment at Seedling Stage
}

\author{
Haiyan Shu, Keming Li, Wei Sun, Guiying Xu, Rulin Zhan*, Shenghe Chang* \\ Haikou Experimental Station, Chinese Academy of Tropical Agricultural Science, Haikou, China \\ Email: *plantfood772@hotmail.com
}

How to cite this paper: Shu, H.Y., Li, K.M., Sun, W., Xu, G.Y., Zhan, R.L. and Chang, S.H. (2019) The Responses of Pineapple Varieties to Forced-Flower Treatment at Seedling. American Journal of Plant Sciences, 10, 2151-2158.

https://doi.org/10.4236/ajps.2019.1012151

Received: November 6, 2019

Accepted: December 7, 2019

Published: December 10, 2019

Copyright $\odot 2019$ by author(s) and Scientific Research Publishing Inc. This work is licensed under the Creative Commons Attribution International License (CC BY 4.0).

http://creativecommons.org/licenses/by/4.0/

\begin{abstract}
Forcing flower is an important step for planting pineapple. Although many papers have been reported about forcing flower for pineapple plants on the aim of harvesting fruits, no reports have been found about forcing flower at seedling phase for preparing bonsai. In this paper, Tainong 4, Tainong 16 and Tainong 17 plants with 20 leaves were watered on stem apex with $40 \mathrm{ml}$ of $0.13 \%$ of ethephron. Results showed that most of Tainong 4 plants flowered after being treated 30 days. None plant of Tainong 16 flowered after being treated 60 days. In the same variety, for example, Tainong 4, some plants flowered earlier, some plants flowered late. Tainong 4 was the most sensitive to ethephron at early-growth phase. Tainong 16 was the most insensitive variety. The low flower rate in this research might be due to that the pineapple plants were in early-vegetative-growth stage. They had not appropriate physiological conditions for forcing flowers. For making pineapple bonsai at early-vegetative-growth phase, higher doses of forcing-flower agent should be used than those of forcing-flower agent for harvesting fruits.
\end{abstract}

\section{Keywords}

Pineapple; Flower, Ethephron, Vegetative-Growth Phase

\section{Introduction}

Pineapple has the largest trade volume among the tropical fruits. Its trade volume is about $50 \%$ of the total volume of tropical fruits [1]. There are about 30 countries planting pineapple in the world. When pineapple plant grows to a certain stage, it can flower naturally under low temperature and shorten day-length [2]-[7]. However, the responses of plants to the same conditions were different, resulting in that some plants flowered and some did not. The flowering times of 
plants are different, leading to low-commodity rate of pineapple fruits. For ensuring all of the plants flowered at the same time, ethephon or calcium carbide is always used to force flowers [8] [9] [10] [11] [12]. After being treated with ethephon or calcium carbide, pineapple plants can flower orderly.

Besides harvesting fruits, pineapple plants can be used as bonsai. When pineapple plants were used as bonsai, long-vegetative-growth phase was not wanted. Pineapple plants with 20 leaves or less can be treated with reagents to force flowers. If the aim was to harvest fruits, pineapple plants with at least 40 leaves are treated. Although many researches have been done about forcing flowers on pineapple, these studies are done on plants for harvesting fruits. For pineapple bonsai, pineapple plants with short-vegetative-growth phase, no papers about forcing flowers have been found. For pineapple plants at seedling stage, how to force flowers with reagents is important for preparing pineapple-bonsai. Pineapple plants (Tainong 4, Tainong 16, Tainong 17) with 20 leaves growing in a pot containing gardening soil were treated with ethephron in this paper. Their responses to ethephron were compared. Plant figures were also observed. The potential application of these responses in making bonsai was also discussed.

\section{Materials and Methods}

Tainong 4, Tainong 16 and Tainong 17 were the main cultivars introduced in recent years. They were used as materials in this paper. After fruits were harvested for one month, suckers whose length was about $30 \mathrm{~cm}$ were collected from mother plants. The suckers were laid in sunlight with the bottom upside for 5 days. On April 20, 2019, suckers of these varieties were planted in pots whose length, width and height were all $80 \mathrm{~cm}$. There was soil in the pots. The formulation of the soil was gardening soil: coconut husk $=1: 1$. The pots were put in the open air in Haikou, China. After one month, nutritional solution was prepared as the following: ammonium nitrate $1650 \mathrm{mg} / \mathrm{L}$, potassium nitrate 1900 $\mathrm{mg} / \mathrm{L}$, monopotassium phosphate $170 \mathrm{mg} / \mathrm{L}$, calcium chloride $440 \mathrm{mg} / \mathrm{L}$, magnesium chloride $370 \mathrm{mg} / \mathrm{L}$. Two hundred of millilitre solution was watered into one pot. The plants were watered one time every two weeks. On September 13, 2019 , three liters of monopotassium phosphate $(0.3 \mathrm{~g} / \mathrm{L})$ were prepared. Three points three three millilitres $(3.33 \mathrm{ml})$ of ethephron $(40 \%)$ were added into the monopotassium phosphate solution. According to the weather forecast, the following four days were all sunny. At zero o'clock at night of September 14, 2019, forty millilitres of forcing-flower solution were watered into the crest of pineapple plant. Twenty plants were treated for each variety. After one month, the plants were observed and the flowers of different varieties were counted.

\section{Results and Discussion}

1) The responses of pineapple varieties to the same dose of ethephron are different

After being treated with $40 \mathrm{ml}$ of $0.13 \%$ of ethephron for one month, most of 
Tainong 4 plants flowered (Figure 1). Neither Tainong 16 nor Tainng17 flowered. After being treated 40 days, in the Tainong 17 plants, only one plant flowered. However, none of Tainong 16 plants flowered (Figure 2). For sensitivity to ethephron, Tainong 4 was the most sensitive variety. Tainong 16 was the most insensitive variety.

2) The responses of different plants of the same variety to ethephron were different

After being treated with ethephron for 30 days, most of Tainong 4 plants flowered. However, one plant of Tainong 4 did not flower until on the $40^{\text {th }}$ day. One plant of Tainong 4 flowered on the $60^{\text {th }}$ day (Figure 3 and Figure 4). For Tainong 17, only one plant flowered on the $40^{\text {th }}$ day (Figure 5). All of the rest plants of Tainong 17 did not flower. These demonstrated that different plants of the same variety had different sensitivity to the same dose of ethephron at early-vegetative-growth phase.

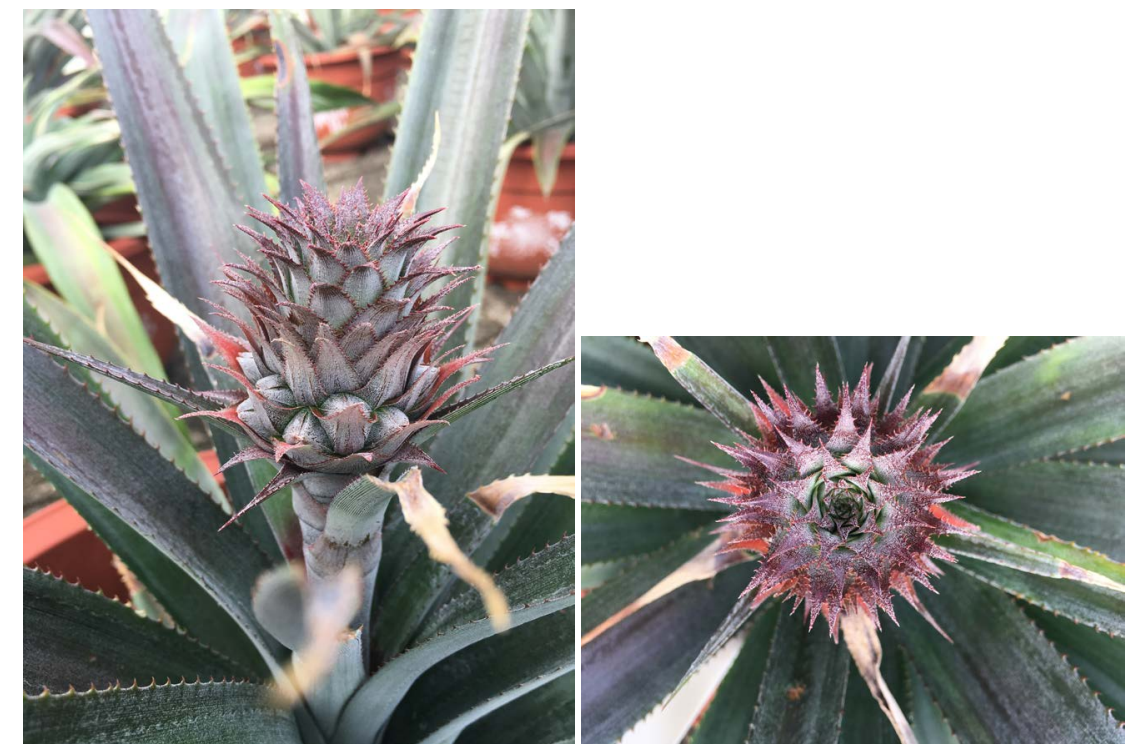

Figure 1. Tainong 4 plants flowered after being treated with ethephron 50 days.

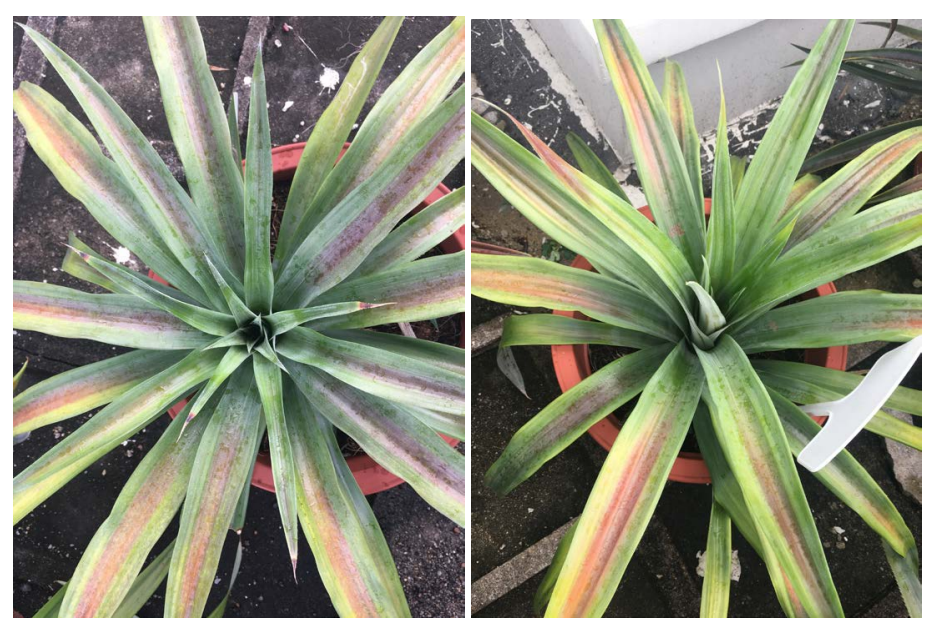

Figure 2. Tainong 16 plants after being treated with ethephron 50 days. 


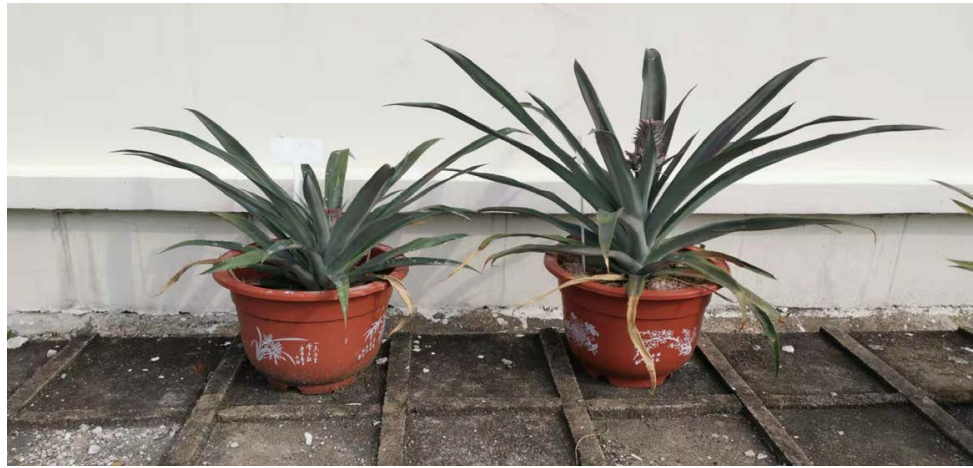

Figure 3. Tainong 4 plants flowered. (The left plant flowered on the $60^{\text {th }}$ day after being treated with ethephron. The right plant flowered on the $30^{\text {th }}$ day after being treated with ethephron.)

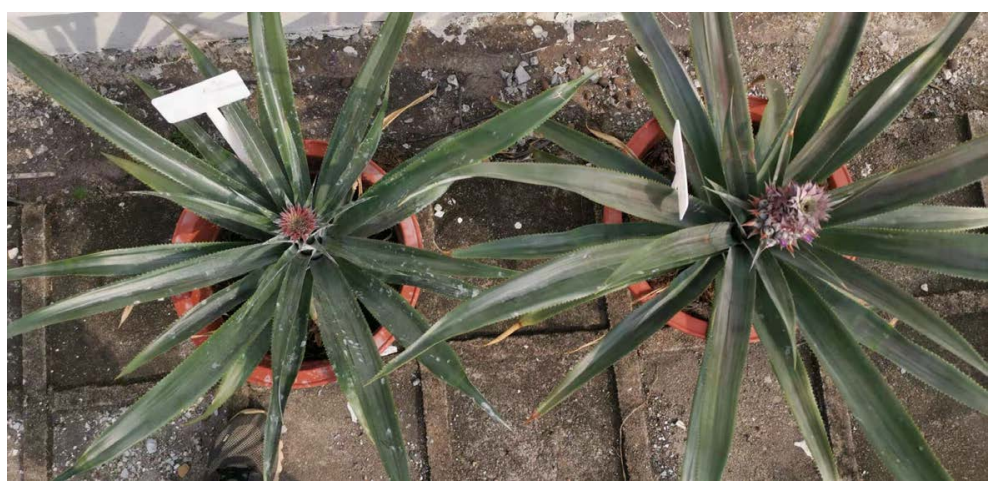

Figure 4. Tainong 4 plants flowered. (The left plant flowered on the $60^{\text {th }}$ day after being treated with ethephron. The right plant flowered on the $30^{\text {th }}$ day after being treated with ethephron.)

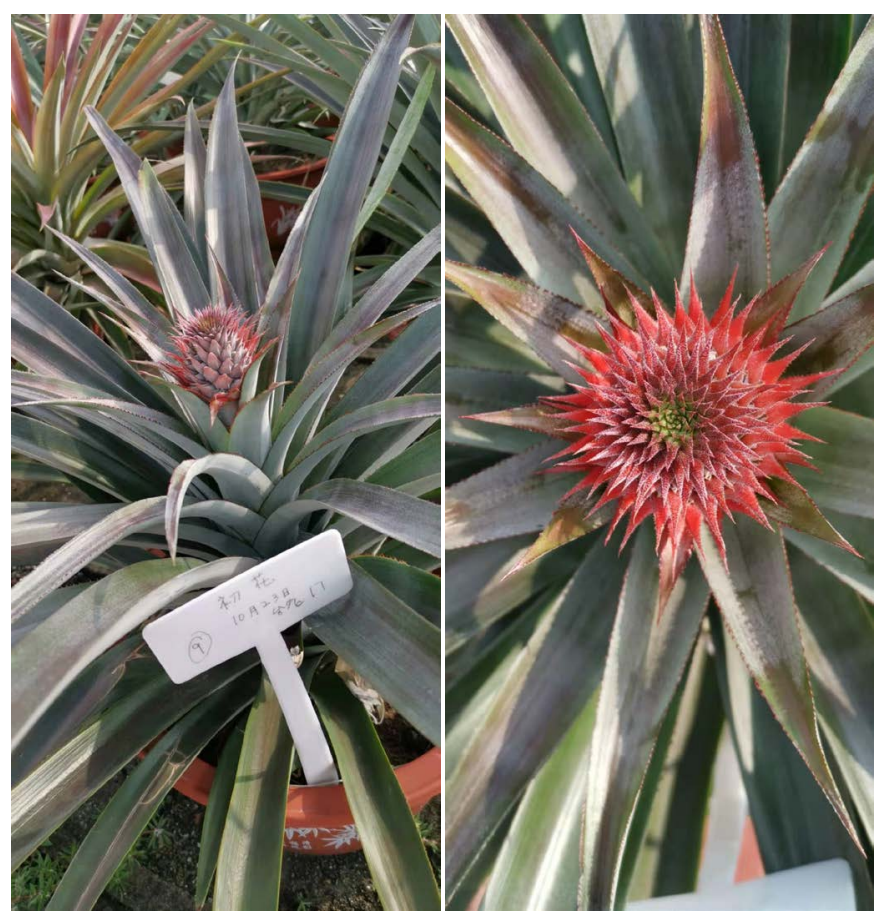

Figure 5. Tainong 17 plants flowered. 


\section{3) The shapes of plants treated with ethephron were different from those} treated with water

After being treated with ethephron, although some plants did not flower, their shapes were significantly different from those treated with water. For Tainong 4 and Tainong 16, fresh leaves of plants treated with ethephron were remarkably shorter than those of plants treated with water (Figures 6-8). The fresh leaves of plants watered with ethephron were more compact than those of plants treated with water (Figures 6-8). Although the similar phenomenon existed in Tainong 17, the differences of the shapes of fresh leaves of Tainong 17 plants watered with ethephron and those of fresh leaves of Tainong 17 plants treated with water were not so much as those in Tainong 4 and Tainong 16 (Figure 9, Figure 10).

The physiological conditions of pineapple plants can affect their sensitivity to forcing-flower reagent. When pineapple plants grew to a certain phase, they will become sensitive to condition factors of inducing flower [13]. For example, pineapple plants at certain growing phase were sensitive to decreasing nutrition

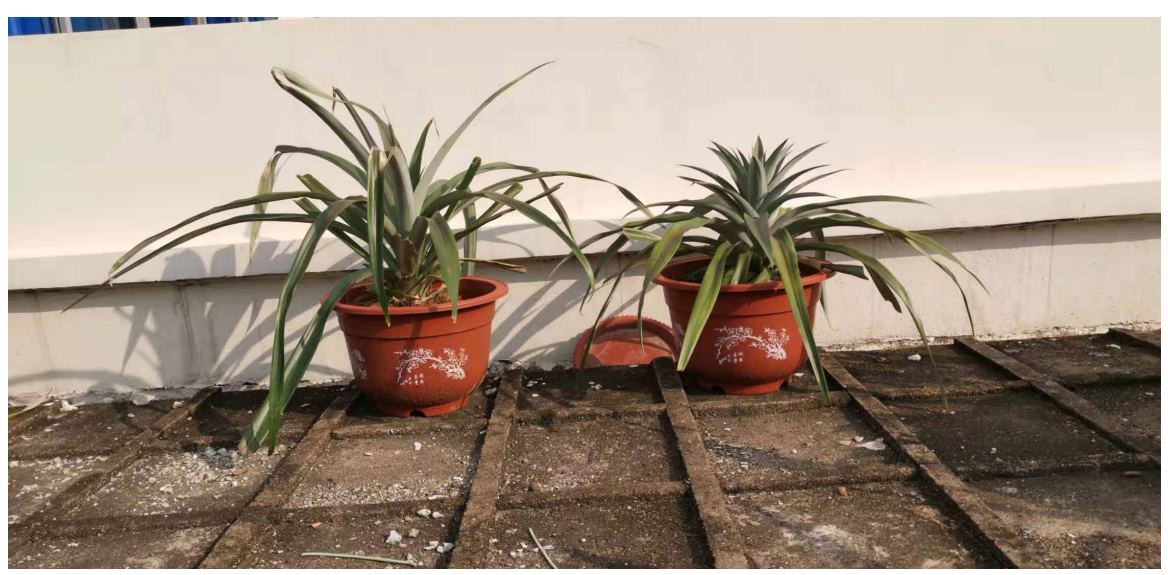

Figure 6. Tainong 16 plants treated with ethephron and plants treated with water. (The left plant was Tainong 16 treated with water. The right plant was Tainong 16 treated with ethephron.)

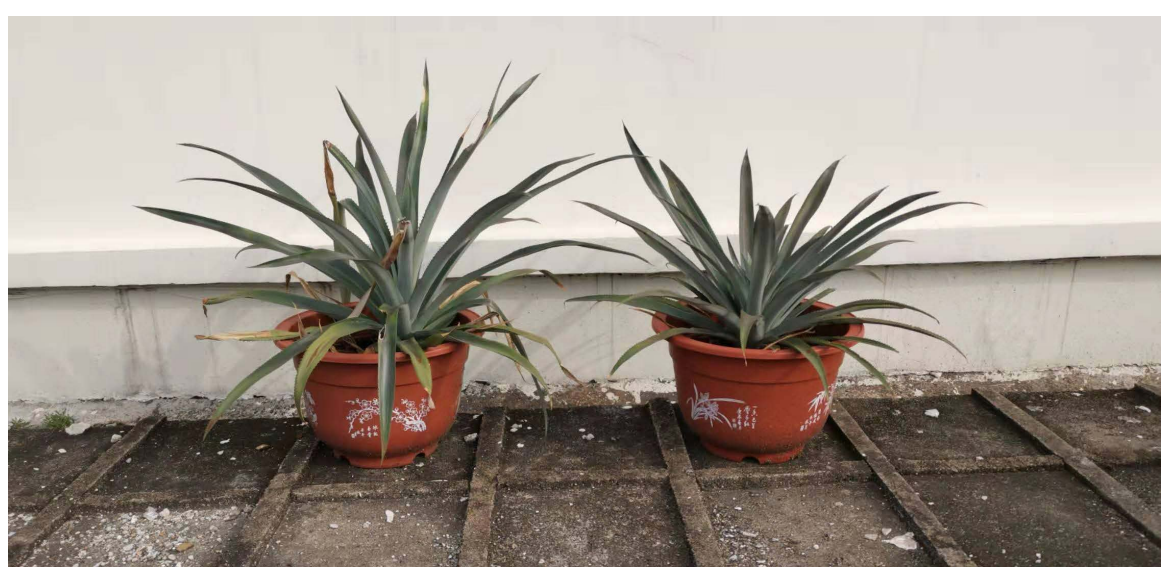

Figure 7. Tainong 4 plants treated with ethephron and plants treated with water. (The left plant was Tainong 4 treated with water. The right plant was Tainong 4 treated with ethephron.) 


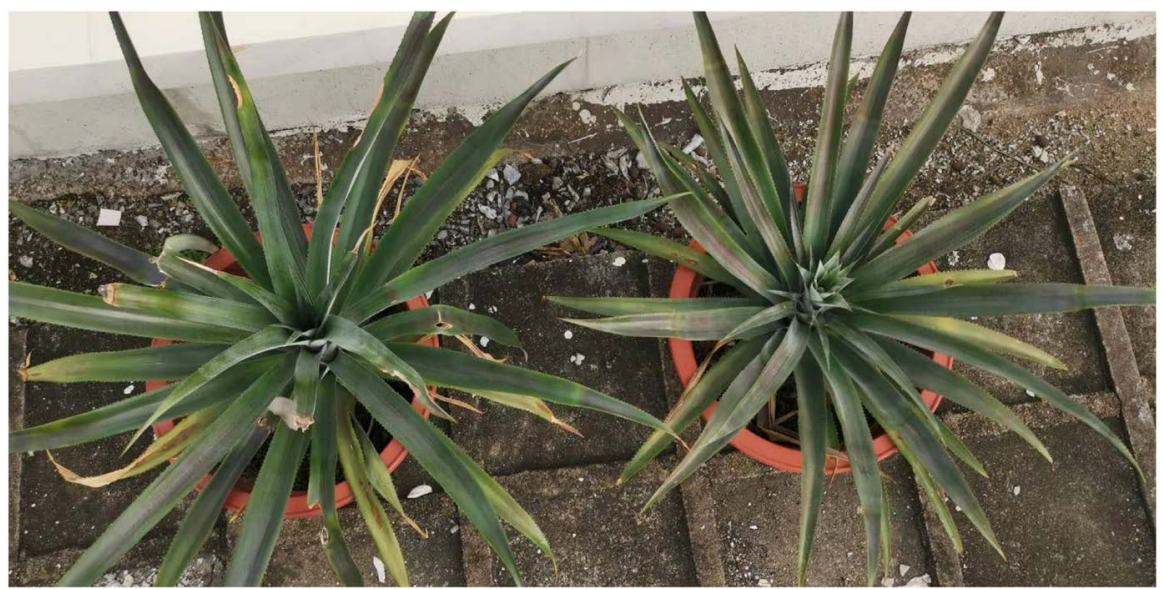

Figure 8. Tainong 4 plants treated with ethephron and plants treated with water. (The left plant was Tainong 4 treated with water. The right plant was Tainong 4 treated with ethephron.)

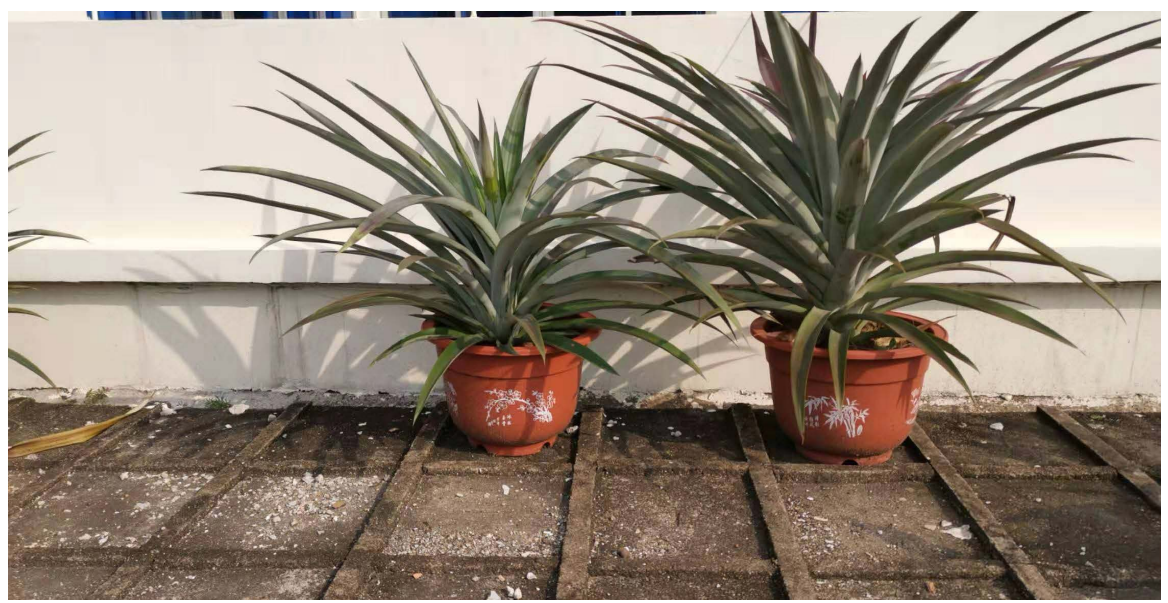

Figure 9. Tainong 17 plants treated with ethephron and plants treated with water. (The left plant was Tainong 17 treated with water. The right plant was Tainong 17 treated with ethephron.)

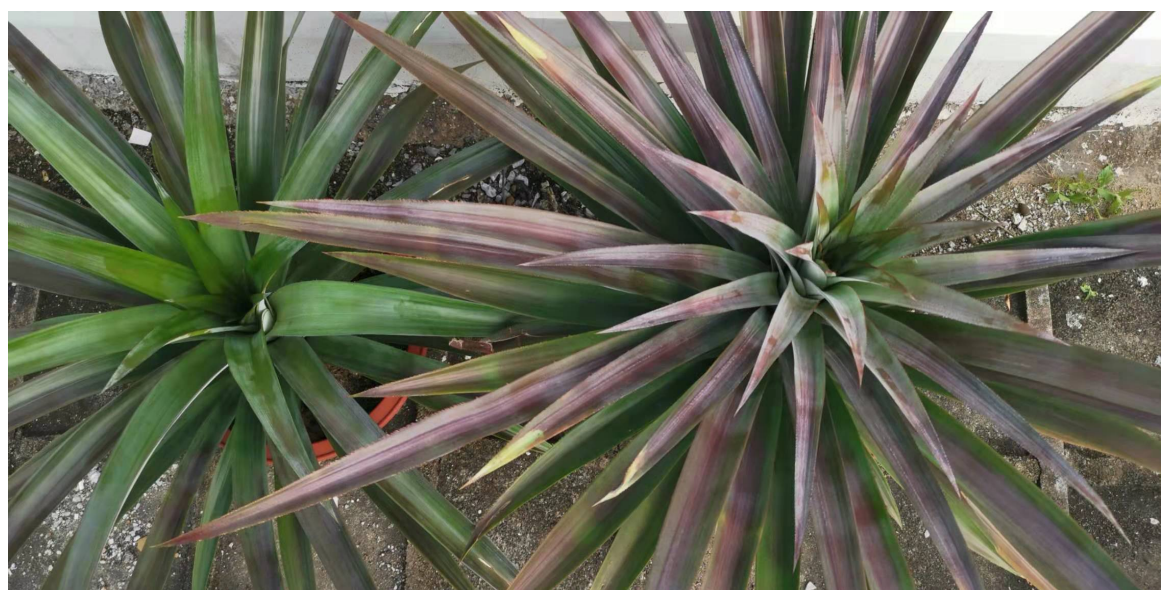

Figure 10. Tainong 17 plants treated with ethephron and plants treated with water. (The left plant was Tainong 17 treated with water. The right plant was Tainong 17 treated with ethephron.) 
and reducing water [2] [14]. Pineapple plants which had not grown to these stages might not be sensitive to these condition factors. In this research, after being treated with $40 \mathrm{ml}$ of $0.13 \%$ of ethephron 60 days, none of Tainong 16 plants flowered. Most of Tainong 17 plants did not flower after being treated with ethephron 60 days. However, after Tainong 16 plants, Tainong 4 and Tainong 17 plants with 40 leaves were watered with the same dose of ethephron 30 days, all of the plants flowered [1]. These demonstrated that none of Tainong 16 plants flowered in this research might be due to that the plants themselves had not grown to a certain stage that fit to be forced flower. They had not appropriate physiological base for reacting to forcing-flower reagents. For preparing pineapple-bonsai using plants at early-growing-vegetative phase, more dose of forcing-flower reagent might be watered.

\section{Acknowledgements}

This paper was supported by the Funds of Ministry of Agriculture and Rural Affairs of the People's Republic of China for Measuring and Controlling the Epidemic Situation of Disease, Pests, and Rats in Crops.

\section{Conflicts of Interest}

The authors declare no conflicts of interest regarding the publication of this paper.

\section{References}

[1] He, J.H., Chen, H.R., Huang, H.J., Wang, J.H. and Huang, H.N. (2015) The New Varieties and High-Quality- and High-Production-Cultivation Technology of Pineapple (Ananas comosus L.). China Agricultural Science and Technology Press, Beijing.

[2] Kuan, C.S., Yu, C.W., Lin, M.L., Hsu, H.T., Bartholomew, D.P., et al. (2005) Foliar Application of Aviglycine Reduces Natural Flowering in Pineapple. HortScience, 40, 123-126. https://doi.org/10.21273/HORTSCI.40.1.123

[3] Maruthasalam, S., Shiu, L.Y., Loganathan, M., Lien, W.C., Liu, Y.L., et al. (2010) Forced Flowering of Pineapple (Ananas comosus cv. Tainon 17) in Response to Cold Stress, Ethephon and Calcium Carbide with or without Activated Charcoal. Plant Growth Regulation, 60, 83-90. https://doi.org/10.1007/s10725-009-9421-9

[4] Van Overbeek, J. and Cruzado, H.J. (1948) Note on Flower Formation in the Pineapple Induced by Low Night Temperatures. Plant Physiology, 23, 282-285. https://doi.org/10.1104/pp.23.3.282

[5] Gowing, D.P. (1961) Experiments on the Photoperiodic Response in Pineapple. American Journal of Botany, 48, 16-21. https://doi.org/10.1002/j.1537-2197.1961.tb11598.x

[6] Friend, D.J.C. and Lydon, J. (1979) Effects of Daylength on Flowering, Growth, and CAM (Crassulacean Acid Metabolism) of Pineapple (Ananas comosus (L.) Merrill). International Journal of Plant Sciences, 140, 280-283.https://doi.org/10.1086/337086

[7] Friend, D.J.C. (1981) Effect of Night Temperature on Flowering and Fruit Size in Pineapple (Ananas comosus (L.) Merr.). International Journal of Plant Sciences, 142, 188-190. https://doi.org/10.1086/337211 
[8] Dass, H.C., Randhawa, G.S., Singh, H.P. and Ganapathy, K.M. (1976) Effect of pH and Urea on the Efficacy of Ethephron for Induction of Flowering in Pineapple. Scientia Horticulturae, 5, 265-268. https://doi.org/10.1016/0304-4238(76)90091-1

[9] Sahoo, A.K., Kar, I., Mohanty, A., Panda, R. and Bhoyar, R.K. (2015) Use of Plant Growth Regulators and Fertilizer for Regulating the Flowering and Quality of Pineapple Fruit: A Review. IJBRITISH, 2, 30-37.

[10] Zhang, J., Yan, C., Liu, Y., Ma, H. and Shi, W. (2013) Effects of Different Forcing Date on Growth of Pineapple Fruit. Hans Journal of Food and Nutrition Science, 2, 25-28. https://doi.org/10.12677/HJFNS.2013.23006

[11] Bartholomew, D.P. and Kadzimin, S.B. (1977) Pineapple. In: Alvim, P.T. and Kozlowski, T.T., Eds., Ecophysiology of Tropical Crops, Academic Press, New York, 113-156. https://doi.org/10.1016/B978-0-12-055650-2.50010-1

[12] Hepton, A. (2003) Cultural System. In: Bartholomew, D.P., Paul, R.E. and Rohrbach, K.G., Eds., The Pineapple: Botany, Production and Use, CABI Publ., Wallingford, 109-142. https://doi.org/10.1079/9780851995038.0109

[13] Van de Poel, B., Ceusters, J. and De Proft, M.P. (2009) Determination of Pineapple (Ananas comosus, MD-2 Hybrid Cultivar) Plant Maturity, the Efficiency of Flowering Induction Agents and the Use of Activated Carbon. Scientia Horticulturae, 120, 58-63. https://doi.org/10.1016/j.scienta.2008.09.014

[14] Lin, M., Chen, A.M., Lin, T., Kuan, C., Lee, C., et al. (2015) Prevention of Natural Flowering in Pineapple (Ananas comosus) by Shading and Urea Application. Horticulture, Environment, and Biotechnology, 56, 9-16.

https://doi.org/10.1007/s13580-015-0095-0 